\title{
A modified digital functional reach test device using an ultrasonic sensor for balance assessment: A test of validity and reliability
}

\author{
Panida Hanphitakphong ${ }^{1}$, Somruthai Poomsalood ${ }^{1}$, Chakkapong Chamroon ${ }^{2}$, \\ Palagon Udomkichpagon ${ }^{1}$
}

${ }^{1}$ Department of Physical Therapy, School of Allied Health Sciences, University of Phayao, Thailand; ${ }^{2}$ Department of Mechanical Engineering, Faculty of Engineering, Chiang Mai University, Thailand

\begin{abstract}
Study aim: Evaluation of dynamic balance is inferred to be compulsory for fall prevention in the elderly. Therefore, this study aimed to develop a modified digital functional reach test device using an ultrasonic sensor for balance assessment and to test validity and reliability of the newly developed tool to qualify psychometric properties.

Material and methods: This study was a cross-sectional study of a convenient sample including 50 participants both males and females. Mean age of the participants was $51.20 \pm 19.30$ years. Reliability of the newly developed device was analysed using the intraclass correlation coefficient (ICC) and standard error of measurement (SEM). The criterion validity was also investigated using a yardstick mounted on the wall at a level of shoulder together with the MaxTraq ${ }^{\circledR} 2 \mathrm{D}$ motion analysis software. The modified digital functional reach test device using an ultrasonic sensor was correlated with the conventional FRT and the MaxTraq ${ }^{\circledR} 2 \mathrm{D}$ motion analysis.

Results: The results presented that test-retest reliability of the modified digital functional reach test device was good reliability $(\mathrm{ICC}=0.76)$ and low standard error of measurement $(1.41)$ was found for test-retest reliability. The degree of agreement between the modified device, the conventional FRT, and the MaxTraq ${ }^{\circledR} 2 \mathrm{D}$ motion analysis was high $(\mathrm{r}=0.71$ and 0.77 respectively).

Conclusions: The findings suggested that the modified digital functional reach test device using an ultrasonic sensor was a valid and reliable instrument for fall risk screening towards functional reach distance.
\end{abstract}

Keywords: Reach distance - Elderly - Ultrasonic sensor - Postural control

\section{Introduction}

The elderly often suffer from slow and progressive decreases in cognitive function [16] and from increasing loss of balance associated with psycho-sensory-motor slowdown [7]. Falls are a major health problem for older individuals and can cause injuries which may lead to disabilities or death [10]. Balance is an important physical characteristic because it is necessary for human motion. Falling is more likely when one's equilibrium is impaired [4, 12]. Fall prevention strategies are most effective if a person at risk is identified before an accident occurs $[1,6,11]$. As a result, clinical balance tests are critical for determining the risk of falling and the effectiveness of balance training. [9].

Standing balance is necessary for everyday tasks' safety and effectiveness. Functional reach test (FRT), first described in the elderly 1990s, is one of several tests of standing balance used in older adults [3]. The FRT is a simple, portable, and clinically approved technique for assessing dynamic balance and determining limits of stability (LOS). It is based on analysing the limits of anteriorposterior stability in the absence of external perturbations. When determining the maximum displacement (FR, distance in $\mathrm{cm}$ ), a subject can achieve in the forward direction without stepping or losing balance. Consequently, it combines biomechanics, postural control, and proprioceptive feedback and correlates results of the higher risk of falling [19]. Previous studies have found that the FRT is a good assessment tool for assessing balance in the elderly in clinical settings. There is evidence for its reliability and validity among the elderly $[8,18]$.

Conventional FRT is used by placing a yardstick or a tape measure on a wall being parallel to the floor at the 
height of the acromion process of an individual's dominant arm. The participant is instructed to stand with the feet at a comfortable distance apart, making a fist, and forwardly flex the dominant arm approximately 90 degrees. The participant is asked to reach forward as far as possible without taking a step or touching the wall. A score is determined by assessing the difference between the starting and ending position of reaching distance [8]. Reach distance of FRT at less than $18.5 \mathrm{~cm}$ has been reported to be associated with the increased risk of falling in the elderly [20].

According to the conventional FRT, limitations of the test can be seen in this single-dynamic task. Firstly, the conventional FRT requires a wall for placing a tape or a yardstick measure. The continuing problem of setting up a tape on the wall, an inconvenience to read values of reach distance as well as data misreading may result from differences in height between individuals. Secondly, FRT can result in the risk of falling especially when an individual reaches forward during the test. This unstable position of the participant can also cause discrepancy when a researcher obtains results on the tape measure as the test needs to be performed twice or three times. Moreover, it is necessary that the researcher is also responsible for safety of the individual during the test. It can be seen that the conventional FRT requires one researcher to multitask that could result in inaccurate data and lack of safety awareness for the elderly due to focusing on reading values on the tape measure.

In clinical settings, there have been several attempts to develop a new instrument to replace the traditional FRT. However, each instrument has its own limitations such as high cost, complicated protocol, and time-consuming machine setup or process $[19,21]$. To overcome these occurrences, a modified instrument that is affordable, movable, adjustable, and easy to use needs to be established. With the increased use of ultrasonic sensors, millimetre range operation with excellent accuracy is now required. An Ultrasonic sensor sends sound pulses towards an object and receives echo signals reflecting from the sensor itself [17]. It is well known that ultrasonic sensors are uncomplicated to use and safe during operation to nearby objects, individuals, or equipment. The common low-cost ultrasonic sensor available on the market is HC-SR04. Due to its ease of construction and low cost, there is evidence that the ultrasonic sensor could be utilised as a digital height metre. Importantly, it provides a touchless and quick method of measuring height [22]. Therefore, the objectives of this study were to develop a modified digital functional reach test device for FRT based on a principle of measuring distance to an object using ultrasonic sound waves and to test validity and reliability of the newly developed tool. The main goal in the tool development was to make it as accurate, user-friendly, and cost-effective as feasible.

\section{Material and methods}

\section{Participants}

This cross-sectional reliability study included 50 participants both males and females aged $18-75$ years. They were recruited following the inclusion criteria: 1) being able to stand and walk at least 3 metres without any ambulatory assistive devices, 2) no history of falls within the past 6 months, 3) not using any orthoses or prostheses, 4) no history of present illness of musculoskeletal system and neuromuscular system at a severe level 5) no history of present illness of neurological system affecting data collection 6) no unresolved visual impairments and 7) being able to communicate and understand instructions. The study was approved by the university research ethics committee (Ethic Code:1.1/007/63). All participants provided written informed consent prior to testing.

\section{Apparatus}

The modified digital FRT instrument for assessing balance was designed using an ultrasonic sensor (HC-SR04) as a measuring device. There are five main parts: 1) distance sensor, which is an ultrasonic sensor (HC-SR04) used to measure distance between a participant's fingertip and the sensor 2) processing unit, which is a microcontroller board (Arduino: UNO R3) used to process a signal from the sensor 3) data storage device, which is a data logger shield for a micro secure digital (SD) card 4) power supply 220VAC to 5VDC and 5) remote control. Data from the processing unit is recorded in the data storage device when a signal from the remote control is active. The mockup was built, as shown in Figure 1, in order to validate reliability of the device.

\section{Balance assessment}

The modified digital FRT device was installed at a level of shoulder on an adjustable tripod. A participant stood facing the device with the feet slightly apart then flexed the shoulder of dominant arm at 90 degrees while wearing a square-shaped plastic sheet on the middle finger of the same arm to reflect the ultrasonic signal. When the participant was in position, a researcher then pressed the remote control to record data at the beginning and read data on the tape measure at the same time. Afterwards, the participant was instructed to reach the arm straight to the sensor as much as possible without making any movement of the feet. The same researcher pressed the remote control to record data and read data on the tape measure when the participant performed the furthest reach. The difference between a distance from the middle fingertip to the sensor before reaching $(\mathrm{X})$ and a distance after reaching $(\mathrm{Y})$ was the reach distance (Figure 2). All data (distance X and Y) were recorded on a micro SD card inserted in the device. 


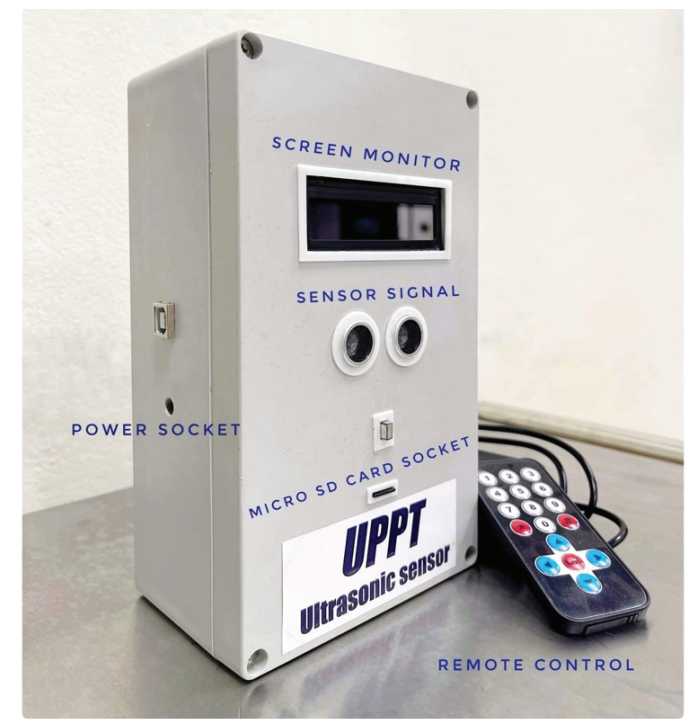

Figure 1. The modified digital FRT device
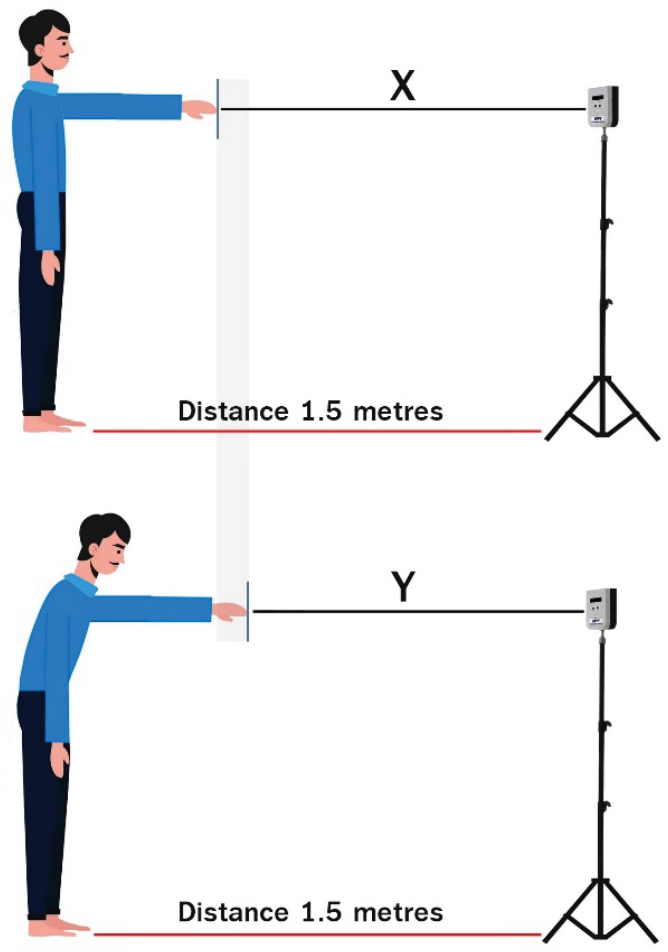

Figure 2. Reach distance: $\mathrm{X}=$ the distance before reaching, $\mathrm{Y}=$ the distance after reaching

The data obtained from the tape measure were recorded on a paper sheet. Each participant performed two trials per session for two sessions. The data recorded on the micro $\mathrm{SD}$ card was presented in millimetres $(\mathrm{mm})$. Alternatively, the distance of $\mathrm{X}$ and $\mathrm{Y}$ was shown on the screen monitor (LCD display) and the data could be manually recorded.

\section{Validity test}

To investigate concurrent validity, balance was assessed two sessions for each participant using the modified FRT device along with the traditional tape measure method. At the same time, another concurrent validity was conducted in 10 out of 50 participants. Balance was examined using the modified FRT device and the traditional tape measure method along with video recording during the test. The reach distance from the video clips was analysed by MaxTraq ${ }^{\circledR} 2 \mathrm{D}$ motion analysis software (Innovision Systems Inc). The software provided raw data at the rate of 30 frames per second (FPS). In this research, Cohen's conventions were used to interpret the effect size. A correlation coefficient of 0.10 is considered to represent a weak or small association, 0.30 considered a moderate correlation, and a correlation coefficient of 0.50 or larger considered to represent a strong or high correlation [5].

\section{Reliability test}

Intra-rater reliability was tested in 30 out of 50 participants by obtaining balance with the modified digital FRT device for 2 times with 5 days apart between visits (testretest reliability). Based on the $95 \%$ confident interval of the ICC estimate, values less than 0.5 , between 0.5 and 0.75 , between 0.75 and 0.9 , and greater than 0.90 are indicated as poor, moderate, good, and excellent reliability respectively [13].

\section{Data analysis}

All data were analysed using SPSS software, version 17.0 for windows. Descriptive statistics was presented as mean and standard deviation (SD). Percentage (as appropriate) was used to calculate baseline characteristic data. Pearson's correlation was used to correlate all instruments that investigated concurrent validity. Intra-rater reliability was examined using the intraclass correlation coefficient. The standard error of measurement (SEM) was calculated as SEM; SD x $\sqrt{ }$ (1-ICC) where SD was the standard deviation of difference score. Dependent (paired) t-test was used to determine a systematic error between Day 1 and Day 2 of testing. Siginificance level of $\alpha=0.05$ was adopted.

\section{Results}

Thirty elderly and twenty young adults (mean age $=51.20 \pm 19.30 \mathrm{yr}$.) completed the protocol (Table 1) Analysis of the concurrent validity and reliability were obtained from mean of reach distance from all measurements. Mean and standard deviation of reach distance are shown in table 2. The modified FRT instrument was useful to evaluate dynamic balance showing strong correlation with the traditional FRT (Pearson's $r=0.71$ ) and the MaxTraq ${ }^{\circledR}$ 2D motion analysis (Pearson's $r=0.77$ ) (Table 3). Intra-class correlation coefficient (ICC) was used to evaluate intra-rater reliability. The results indicated the modified 
Table 1. Demographic characteristics of participants $(\mathrm{N}=50$; males $=22$, females $=28)$

\begin{tabular}{lcccc}
\hline Data & Mean \pm SD & Minimum & Maximum & Range \\
\hline Age $[\mathrm{yr}]$ & $51.20 \pm 19.30$ & 22 & 73 & 51 \\
Weight $[\mathrm{kg}]$ & $56.48 \pm 5.93$ & 44.00 & 68.50 & 24.50 \\
Height $[\mathrm{cm}]$ & $163.08 \pm 6.06$ & 150.00 & 175.00 & 25.00 \\
BMI $\left[\mathrm{kg} / \mathrm{m}^{2}\right]$ & $21.20 \pm 1.42$ & 18.64 & 22.99 & 4.35 \\
\hline
\end{tabular}

Table 2. Mean \pm standard deviation of reach distance in each measurement

\begin{tabular}{lc}
\hline Measurement & Reach distance $[\mathrm{cm}]$ \\
\hline Conventional FRT $(\mathrm{N}=50)$ & $23.92 \pm 5.29$ \\
The modified digital FRT device $(\mathrm{N}=50)$ & $23.81 \pm 5.40$ \\
MaxTraq ${ }^{\circledR}$ 2D motion analysis $(\mathrm{N}=10)$ & $26.87 \pm 7.68$ \\
\hline
\end{tabular}

Table 3. Pearson correlation coefficient between different measurements

\begin{tabular}{lcccc}
\hline \multirow{2}{*}{ Measurement } & \multicolumn{2}{c}{ Conventional FRT $(\mathrm{N}=50)$} & MaxTraq® 2D motion analysis $(\mathrm{N}=10)$ \\
\cline { 2 - 4 } & $\mathrm{r}$ & $p$-value & $\mathrm{r}$ & $p$-value \\
\hline $\begin{array}{l}\text { The modified digital FRT } \\
\text { device }(\mathrm{N}=50)\end{array}$ & 0.71 & $<0.001^{*}$ & 0.77 & $0.009^{*}$ \\
\hline
\end{tabular}

*Significant at $p \leq 0.05$ (two-tailed).

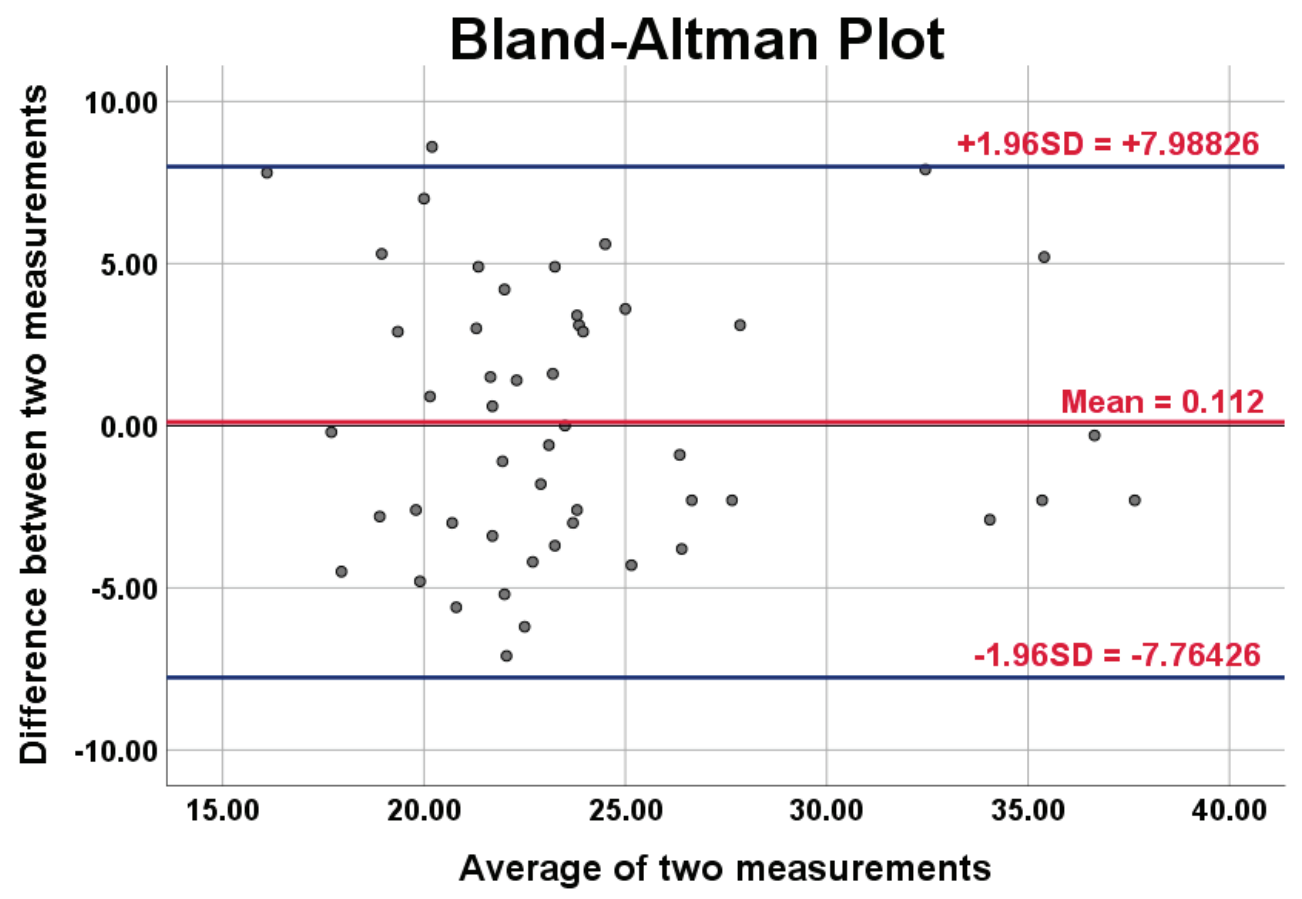

Figure 3. Bland-Altman plot of reach distance: the red line represents mean difference between two measurements and the blue lines represent $95 \%$ limits of agreement 
Table 4. Intra-rater reliability of the modified digital FRT device $(\mathrm{N}=30)$

\begin{tabular}{ccccccccc}
\hline & \multicolumn{2}{c}{ Session } & \multicolumn{3}{c}{ Test-retest reliability } & \multicolumn{2}{c}{ Paired differences } \\
\cline { 2 - 8 } & Day 1 & Day 2 & ICC & SEM & $\begin{array}{c}p \text {-value } \\
(95 \% \text { CI })\end{array}$ & $\begin{array}{c}\text { Mean } \\
\text { difference }\end{array}$ & $\begin{array}{c}p \text {-value } \\
(95 \% \text { CI })\end{array}$ \\
\hline Distance [cm] & $22.08 \pm 2.93$ & $22.92 \pm 3.55$ & 0.76 & 1.41 & $\begin{array}{c}<0.001 * \\
(0.50 \text { to } 0.89)\end{array}$ & 0.85 & $\begin{array}{c}0.118 \\
(0.50 \text { to } 0.89)\end{array}$ \\
\hline
\end{tabular}

*Significant at $p \leq 0.05$.

FRT instrument provided high reliability of intra-rater reliability $(\mathrm{ICC}=0.76)$; $\mathrm{SEM}=1.41 \mathrm{~cm}$. Considering paired t-test, no significant difference was found between Day 1 and Day 2 of testing (Table 4). As a result of the BlandAltman analysis, no fixed bias was observed because upper and lower limits of $95 \%$ CI of the difference between the 2 measurements in all measurement outcomes were all values that zero was considered (Figure 3 ).

\section{Discussion}

In the present study, the authors employed a principlebased ultrasonic sensor to develop the modified digital functional reach test device. The results of this study showed that the modified digital functional reach test device using an ultrasonic sensor presented with good test-retest reliability and concurrent validity. In this study, measurement of functional reach test demonstrated high reliability with ICC of 0.76 being in line with the result shown by Duncan et al. (1990) [8] who used the yard stick. Regarding SEM, it is directly related to reliability of test. Generally, larger SEM represents lower reliability of test [14]. In this study, SEM; 1.41 indicated an acceptable level of reliability. Moreover, paired t-test was used to determine the systematic error from repeatability. Systematic errors can be identified by means of several statistical tests, such as paired t-test, the intraclass correlation, and the Bland-Altman method. The finding of paired t-test suggested that the modified device indicated an absence of systematic error due to unrevealed statistically significant systematic error. Regarding validity, a high correlation found between the modified digital functional reach test device and the conventional FRT $(\mathrm{r}=0.71)$ indicated that both tools can interchange to utilise. According to this study, the modified digital functional reach test device may be recommended for measuring forward reach distance as it was also correlated with the MaxTraq ${ }^{\circledR} 2 \mathrm{D}$ motion analysis $(r=0.77)$. However, only a small sample was conducted and analysed by the MaxTraq ${ }^{\circledR} 2 \mathrm{D}$ motion analysis software.

It is difficult to compare the results of the present study to other studies due to different tools. The conventional FRT is one of the most common tools in clinical practice as it is simple, easy, and requires minimum time to administrate $[15,19,21]$. Additionally, it provides an accurate measure to assess balance in the elderly [2, 19]. Although only single-dynamic task is required, the test is susceptibility to fall enhanced by reach task during assessment. The modified digital functional reach test device using an ultrasonic sensor was invented by the authors and considered to be more convenient and practical to be used by older adult participants who require safety guarding. The conventional FRT is measured by an observer with reference to a graduated tape measure and does not require any specific equipment. The observer needs to stand very close to a subject and tape measure for determining the value of test and providing safety testing for the subject. For the conventional FRT, the scale is read at the level of the observer's eyes which could cause an error from reading and estimation. Unlike the FRT, this newly modified device can be used without a tape measure and smooth wall by utilising the sensor. It is easy to be mobilised, adjusted for height, and assembled in any place. Importantly, it is less time-consuming and very helpful for data analysis with a mini SD card.

Some limitations were found in this study. Firstly, only healthy subjects were recruited as the sample group. All participants did not present with any conditions that affected balance and falling except for increasing of age which means that the results of the present study cannot be generalised for all individuals. Importantly, all participants reported no falls in the previous 6 months. According to the guideline, reach distance of FRT at less than $18.5 \mathrm{~cm}$ has been reported to be associated with the increased risk of falling in the elderly [20]. In this study, the average values of reach distance among the three techniques were between $23.81 \pm 5.40$ to $26.87 \pm 7.68 \mathrm{~cm}$ which were greater than the cutoff value. This showed that all the volunteers had no condition related to falls or impaired balance. Further investigation in individuals with impaired balance or specific conditions such as obese diabetic patients, individuals with dementia, stroke patients, and hypertensive older adults are required. Secondly, the authors intended to develop a device to assess the risk of falling in the elderly, but 20 younger participants were also recruited in the 
process of tool quality assessment. However, when considering the number of the elderly in this study which was 30 , it can be seen that the amount was more than $50 \%$ of the total participants which was sufficient for quality assessment of the tool. In the authors' perspective, recruiting younger volunteers may benefit the equipment use in other cases that do not result from aging such as individuals with balance impairment from general or sports injuries or from neurological disorders. Thirdly, there was a lack of inter-rater reliability test due to a limited number of researchers who participated in this current study. Further research needs to be obtained to fulfil comprehensive reliability. Fourthly, temperature change might affect the accuracy of distance measurement. However, the researchers attempted to control room temperature of the experimental laboratory throughout the testing process. Thus, it would be beneficial in future research to use an ultrasonic sensor in combination with a temperature sensor. Another limitation was that responsiveness of reach distance measured by the modified digital functional reach test device, which is crucial for clinicians to assess and treat their patients, was not investigated in this study. Testing of responsiveness should be conducted in a longitudinal study in individuals with balance impairment as well as the risk of falling. Moreover, it would be more useful to investigate reach distance with respect to multi-direction in future studies along with having experts assess and confirm the validity of the research tool.

\section{Conclusions}

Theoretically, balance is required for maintaining static posture and for stabilising dynamic movements. Therefore, there is no best method to measure balance with comprehensive aspects. In general, there are several balance assessing methods which can be applied together, such as Berg Balance Scale (BBS), Functional Reach Test (FRT), and Timed Up and Go test (TUG).

As mentioned above, the conventional FRT is one of the tools that is most widely used in clinical settings. It is well accepted due to its good validity and reliability which can assess dynamic balance and indirectly measures limits of stability. However, the modified digital functional reach test device is necessary to eliminate limitations of the conventional FRT. Therefore, the good positive correlation between reach distance measured by the modified digital functional reach test device, the conventional FRT, and the MaxTraq ${ }^{\circledR}$ 2D motion analysis as demonstrated in the present study supports the recommendation that the modified digital functional reach test device is a valid and reliable measurement tool that can be used to measure functional reach distance in adults and the elderly.

\section{Clinical implications}

Clinicians always attempt to prevent falls in older adults by various strategy approach. In order to screen the risk of falling, plan, and assess the effectiveness of interventions, it is vital to develop an objective method to measure balance and fall risk. Considering implement for clinical use, the newly modified device is practical, userfriendly, reliable. Based on this study, the modified digital functional reach test device is an inexpensive, portable, and convenient apparatus that allows clinicians to collect accurate, reliable, and objective reading.

\section{Conflict of interest: Authors state no conflict of interest.}

\section{References}

1. Allen B., Derveloy R., Lowry K., Handley H., Fell N., Gasior W., Yu G., Sartipi M. (2013) Evaluation of fall risk for post-stroke patients using bluetooth low-energy wireless sensor. IEEE Global Communications Conference (GLOBECOM), 2598-2603.

2. Behrman A.L., Light K.E., Flynn S.M., Thigpen M.T. (2002) Is the functional reach test useful for identifying falls risk among individuals with Parkinson's disease? Arch. Phys. Med. Rehabil., 83(4): 538-542. DOI: 10.1053/apmr.2002.30934.

3. Bohannon R.W., Wolfson L.I., White W.B. (2017) Functional reach of older adults: normative reference values based on new and published data. Physiotherapy, 103(4): 387-391. DOI: 10.1016/j.physio.2017.03.006.

4. Bouaziz W., Lang P.O., Schmitt E., Kaltenbach G., Geny B., Vogel T. (2016) Health benefits of multicomponent training programmes in seniors: a systematic review. Int. J. Clin. Pract., 70(7): 520-536. DOI: 10.1111/ ijcp. 12822 .

5. Cohen J. (1988) Statistical power analysis (2nd ed.). Routledge, New York. DOI: 10.4324/9780203771587.

6. Danielsen A., Olofsen H., Bremdal B.A. (2016) Increasing fall risk awareness using wearables: a fall risk awareness protocol. J. Biomed. Inform., 63: 184-194. DOI: 10.1016/j.jbi.2016.08.016.

7. Deshpande N., Metter E.J., Ferrucci L. (2011) Sensorymotor and psychosocial correlates of adaptive locomotor performance in older adults. Arch. Phys. Med. Rehabil., 92(7): 1074-1079. DOI:10.1016/j.apmr.2011.02.006.

8. Duncan P.W., Weiner D.K., Chandler J., Studenski S. (1990) Functional reach: a new clinical measure of balance. J. Gerontol., 45(6): 192-197. DOI: 10.1093/ geronj/45.6.m192.

9. Freeman J., Fox E., Gear M., Hough A. (2012) Pilates based core stability training in ambulant individuals with multiple sclerosis: protocol for a multi-centre ran- 
domised controlled trial. BMC Neurol., 5: 12-19. DOI: 10.1186/1471-2377-12-19.

10. Guirguis-Blake J.M., Michael Y.L., Perdue L.A., Coppola E.L., Beil T.L. (2018) Interventions to prevent falls in older adults updated evidence report and systematic review for the US preventive services task force. JAMA, 319(16):1705-1716. DOI:10.1001/jama.2017.21962.

11. Hahn M.E., Chou L.S. (2003) Can motion of individual body segments identify dynamic instability in the elderly? Clin. Biomech. (Bristol, Avon), 18(8): 737-744. DOI: 10.1016/s0268-0033(03)00139-6.

12. Han J., Anson J., Waddington G., Adams R., Liu Y. (2015) The role of ankle proprioception for balance control in relation to sports performance and injury. Biomed. Res. Int., 2015: 842804. DOI: 10.1155/2015/842804.

13. Koo T.K., Li M.Y. (2016) A guideline of selecting and reporting intraclass correlation coefficients for reliability research. J. Chiropr. Med., 15(2): 155-163. DOI: 10.1016/j.jcm.2016.02.012.

14. Matheson G.J. (2019) We need to talk about reliability: making better use of test-retest studies for study design and interpretation. PeerJ., 7: e6918. DOI: 10.7717/peerj.6918.

15. Merchán-Baeza J.A., González-Sánchez M., CuestaVargas A.I. (2014) Reliability in the parameterization of the functional reach test in elderly stroke patients: a pilot study. Biomed. Res. Int., 2014, Article ID 637671, 8 pages. DOI: $10.1155 / 2014 / 637671$.

16. Okonkwo O.C., Griffith H.R., Copeland J.N., Belue K., Lanza S., Zamrini E.Y., Harrell L.E., Brockington J.C., Clark D., Raman R., Marson D.C. (2008) Medical decision-making capacity in mild cognitive impairment: a 3-year longitudinal study. Neurology, 71(19): 1474-1480. DOI: 10.1212/01.wnl.0000334301.32358.48.
17. Panda K.G., Agrawal D., Nshimiyimana A., Hossain A. (2016) Effects of environment on accuracy of ultrasonic sensor operates in millimetre range. Perspect. Sci., 8: 574-576. DOI: 10.1016/j.pisc.2016.06.024.

18. Rosa M.V., Perracini M.R., Ricci N.A. (2019) Usefulness, assessment and normative data of the functional reach test in older adults: a systematic review and meta-analysis. Arch. Gerontol. Geriatr., 81:149-170. DOI: 10.1016/j.archger.2018.11.015.

19. Scena S., Steindler R., Ceci M., Zuccaro S.M., Carmeli E. (2016) Computerized functional reach test to measure balance stability in elderly patients with neurological disorders. J. Clin. Med. Res., 8(10): 715-720. DOI: 10.14740/jocmr2652w.

20. Thomas J.I., Lane J.V. (2005) A pilot study to explore the predictive validity of 4 measures of falls risk in frail elderly patients. Arch. Phys. Med. Rehabil., 86(8): 1636-1640. DOI: 10.1016/j.apmr.2005.03.004.

21. Williams B., Allen B., Hu Z., True H., Cho J., Harris A., Fell N., Sartipi M. (2017) Real-time fall risk assessment using functional reach test. Int. J. Telemed. Appl., 2017: 2042974. DOI: 10.1155/2017/2042974.

22. Wongke T., Awikunprasert P., Awikunprasert C. (2017) Digital height meter using ultrasonic sensor. J. Assoc. Med. Sci., 50(3): 435-441.

\section{Received 07.06.2021 \\ Accepted 22.07.2021}

(C) University of Physical Education, Warsaw, Poland 